\title{
Determining true North on Mars by using a sundial on InSight
}

\author{
D. Savoie ${ }^{\mathrm{a}, *}$, A. Richard ${ }^{\mathrm{b}, * *}$, M. Goutaudier ${ }^{\mathrm{b}}$, N.P. Onufer ${ }^{\mathrm{c}}$, M.C. Wallace ${ }^{\mathrm{c}}$, D. Mimoun ${ }^{\mathrm{e}}$,

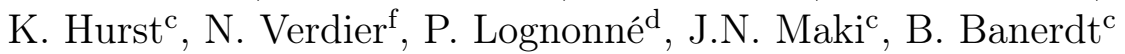 \\ ${ }^{a}$ SYRTE, Observatoire de Paris, Université PSL, CNRS, Sorbonne Université, LNE, 61 avenue de l'Observatoire \\ 75014 Paris, France \\ ${ }^{b}$ Palais de la Découverte, Av. Franklin D. Roosevelt, 75008 Paris, France \\ ${ }^{c}$ NASA Jet Propulsion Laboratory, Pasadena, California \\ ${ }^{d}$ Institut de Physique du Globe de Paris, Université Paris Diderot, Paris, France \\ ${ }^{e}$ Institut Supérieur de l'Aéronautique et de l'Espace, ISAE, Toulouse, France \\ ${ }^{f}$ French National Space Agency, CNES, Paris, France
}

\begin{abstract}
In this work, we demonstrate the possibility to determine the true North direction on Mars by using a gnomon on the InSight mission. The Sun local coordinates are computed using the analytical solution VSOP87 for the planetary ephemeris. The Sun position in the sky induce specific gnomon shadow orientation and length that can be read on a target. By comparing the images of the shadow with the expected position of the Sun, we can determine the true North direction with an accuracy up to $1^{\circ}$, well within the seismic requirement of $5^{\circ}$, even if the lander position or the gnomon verticality are not accurate. To achieve this result, we determine the best periods of observation during the SEIS deployment phase in December 2018. A total period of 5 hours has been identified on mornings and evenings on Mars, during which the impact of errors on position and verticality on the true North direction are minimised.
\end{abstract}

NASA's InSight landing on Mars is scheduled for November $2018^{1}$. The space probe has among its instruments the seismometer SEIS (Seismic Experience for Interior Structure) (Lognonné et al., 2011), a single seismic station that will be deployed on a planet on which no model of seismic propagation waves has ever been made by previous missions. See Lognonné et al. (2018) for a complete description of the SEIS experiment and Lognonné and Johnson (2015) for review on planetary seismology, including Mars.

The measurement of the azimuth of seismic waves is offering a direct measurement of the propagation of seismic waves: for body waves, $\mathrm{P}$ compressional waves are indeed longitudinal while $\mathrm{S}$ waves are transverse and the same properties applied for surface waves and for Rayleigh and Love waves respectively. Measuring these features through the determination of the back-azimuth of the waves in 3 axis seismograms is therefore one of the first processing made on seismograms, just

\footnotetext{
${ }^{*}$ Corresponding author

${ }^{* *}$ Principal corresponding author

Email addresses: savoie@obspm.fr (D. Savoie), andy.richard@universcience.fr (A. Richard), marc.goutaudier@universcience.fr (M. Goutaudier)

${ }^{1}$ https://mars.nasa.gov/insight/mission/overview/
} 
after the arrival time determination (e.g. Bormann et al. (2009)). This back-azimuth is therefore key in all single station location technics, either used on Earth (e.g. Braak (1910) and many works in the following century) or planned on Mars for InSIght (Panning et al., 2015; Khan et al., 2016; Böse et al., 2017) and given in more detail by Clinton et al. (2018). In addition and for signals suspected to be associated to impacts, the back-azimuth will provide together with an estimated epicentral distance obtained from either differential $\mathrm{P}$ and $\mathrm{S}$ arrival time or $\mathrm{P}$ and Surface waves differential arrival time, the possibility to request orbiters to map the Mars surface for determining the exact position of the fresh impacts (see Daubar et al. (2018) for more details). The same will be requested to understand the signature of dust devils and of wind generated noise (e.g. Kenda et al. (2017)), as both might generate surface features documented by high resolution imaging (e.g. Reiss et al. (2016) for a review).

The precise knowledge of the azimuth of the three oblique axes of the Very Broad Band sensor (VBB) and two horizontal Short Period sensor (SP) is therefore mandatory to achieve the location of quakes and impacts and this knowledge was required to be known better than $5^{\circ}$. The SEIS experiment is equipped with a handle used by InSight to deploy the experiment on the surface. This handle will also be used as a gnomon. A gnomon is the main tool of a sundial: a vertical stick projecting a shadow on a target which enables to determine the Sun position in the sky, and thus by extension the seismometer position and orientation on Mars surface. The SEIS sundial will provide a secondary measurement, in addition to the one made by the Inertial Measurement Unit (IMU) just after the landing. The IMU will define the precise orientation of the lander too and can therefore be used for extrapolating the one of SEIS from the High resolution pictures taken by InSight cameras (Maki et al., 2018).

In this paper, we describe the method used to determine the true North direction by using the SEIS gnomon. In the first part, we compute the Sun coordinates for an observer located on Mars. Those coordinates are required to provide indirectly the size and location of the shadow of the gnomon on the target. Then in a second part, we describe the gnomon and the target used on SEIS to determine the Sun position. We also describe the method used to extract the orientation of SEIS from images taken by InSight. Finally, we quantify the different errors that can affect the true North determination.

\section{Solar coordinates for a Martian observer}

The position of the shadow of the gnomon over the seismometer SEIS is determined by the Sun position in the sky for a Martian observer. In this paper, we investigate the case of the Sun local coordinates at the InSight expected landing site in Elysium Planitia (Golombek et al., 2017) during December 2018, the first images of the sundial are scheduled for December 20.

\subsection{Transformation to the areocentric coordinates system}

To obtain the heliocentric coordinates of Mars, we use the ephemeris obtained by the analytical planetary solution VSOP87 (Bretagnon, 1982; Bretagnon and Francou, 1988). VSOP87 is a theory for the motion of the eight planets based on a Poisson series expansion of the solutions. VSOP87 is not the last version available of the VSOP solution, but its accuracy for Mars of $1^{\prime \prime}$ over 4000 years after J2000 is sufficient in the case of this study.

For any given date, the VSOP87 solution provides the true position vector $\vec{r}_{p}{ }^{\prime}$ of Mars in the heliocentric reference frame $\left(O_{S}, X^{\prime}, Y^{\prime}, Z^{\prime}\right)$ associated to the mean ecliptic at J2000. Thus, 
the Sun true position vector $\vec{r}_{s}{ }^{\prime}$ in the areocentric reference frame $\left(O, X^{\prime}, Y^{\prime}, Z^{\prime}\right)$ is given by a translation from the Sun center to Mars center.

\subsubsection{Mars orientation parameters according to Konopliv et al. (2006, 2016)}

Determination of the Sun local coordinates for a Martian observer requires to express the vector $\vec{r}_{s}{ }^{\prime}$ in a reference frame associated to the Martian equator and based on a prime meridian. The prime meridian for planets is defined by IAU (e.g. Archinal et al. (2011)) and based on observable fixed surface features. To do so, Mars orientation with respect to the mean ecliptic J2000 reference frame is needed. The global solution for Mars orientation provided by Konopliv et al. (2006, 2016) is based on the tracking data collection from Mars Global Surveyor, Mars Reconnaissance Orbiter or MER Opportunity, and other data sets from Mars Odyssey, Mars Pathfinder or Viking Lander. Using the values of the different angles, we can transform the coordinates from the mean ecliptic J2000 reference frame to the Mars true equator of date reference frame (Figure 1).

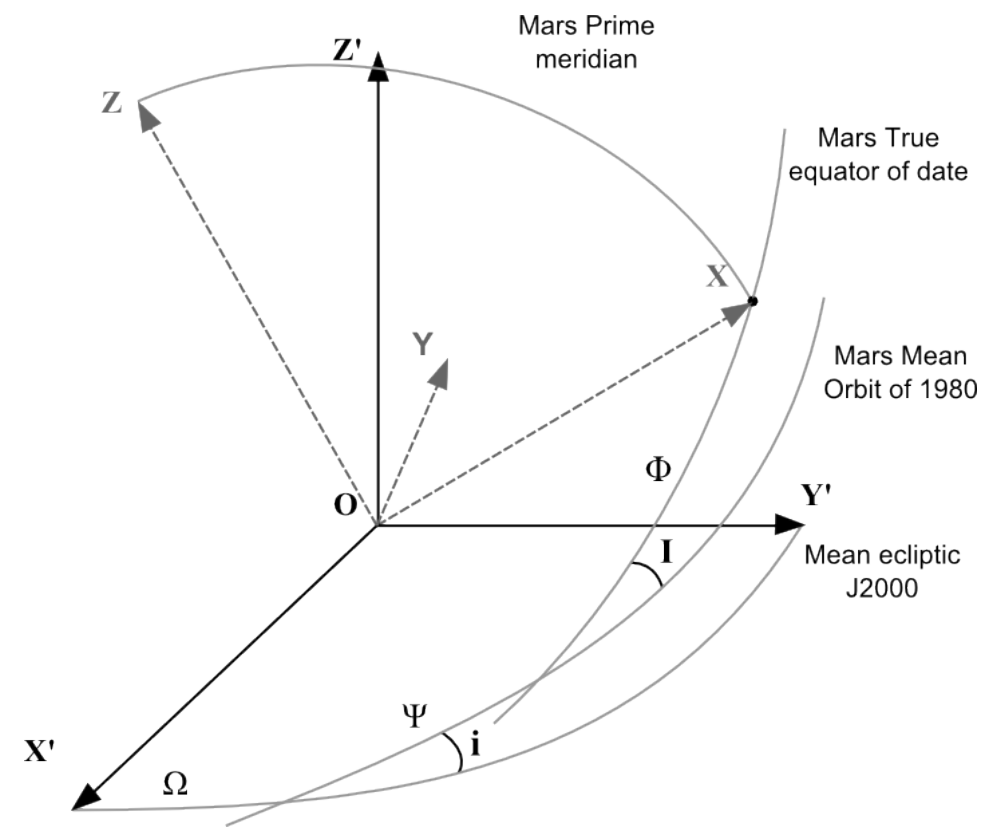

Figure 1: Mars orientation parameters as expressed in Konopliv et al. (2006)

The Sun position vector $\vec{r}_{s}$ in the Martian true equator of date reference frame $(O, X, Y, Z)$ and based on the prime meridian is given by:

$$
\vec{r}_{s}=R_{z}(\Phi) R_{x}(I) R_{z}(\Psi) R_{x}(i) R_{z}(\Omega) \vec{r}_{s}{ }^{\prime},
$$

where $R_{x}$ and $R_{z}$ are the rotation matrices defined as

$$
\begin{aligned}
& R_{x}(\theta)=\left(\begin{array}{ccc}
1 & 0 & 0 \\
0 & \cos \theta & \sin \theta \\
0 & -\sin \theta & \cos \theta
\end{array}\right) \\
& R_{z}(\theta)=\left(\begin{array}{ccc}
\cos \theta & \sin \theta & 0 \\
-\sin \theta & \cos \theta & 0 \\
0 & 0 & 1
\end{array}\right) .
\end{aligned}
$$


$\Omega$ is the longitude of the ascending node of Mars orbit at J1980, $i$ is the inclination of Mars orbit at J1980, I is Mars obliquity with respect to the orbital plane at J1980, $\Psi$ is Mars true equator longitude with respect to the orbital plane J1980, and $\Phi$ is the prime meridian longitude. The new reference frame $(O, X, Y, Z)$ is thus oriented according to the Martian prime meridian and the Martian true North direction. The numerical values of the different angles are given in Table $1 . \Psi$ and $I$ are also affected by precession and nutation such as

$$
\begin{aligned}
\Psi(t) & =\Psi_{o}+\dot{\Psi}_{o} t+\Psi_{n u t}, \\
I(t) & =I_{o}+\dot{I}_{o} t+I_{n u t},
\end{aligned}
$$

where the components due to nutation $\Psi_{n u t}$ and $I_{n u t}$ are expressed as series with coefficients given by Konopliv et al. (2006).

\begin{tabular}{lll}
\hline \multicolumn{1}{c}{ Parameter } & Value & Source \\
\hline$\Omega(\mathrm{deg})$ & 49.6167 & Konopliv et al. $(2006)$ \\
$i(\mathrm{deg})$ & 1.85137 & Konopliv et al. $(2006)^{*}$ \\
$\Psi_{o}(\mathrm{deg})$ & 81.9683988 & Konopliv et al. $(2016)$ \\
$\dot{\Psi}_{o}(\mathrm{mas} /$ year $)$ & -7608.3 & Konopliv et al. $(2016)$ \\
$I_{o}(\mathrm{deg})$ & 25.1893823 & Konopliv et al. $(2016)$ \\
$\dot{I}_{o}(\mathrm{mas} /$ year $)$ & -2.0 & Konopliv et al. $(2016)$ \\
& & \\
\hline
\end{tabular}

*A mistake has been corrected here

for the inclination value.

Table 1: Mars orientation parameters

\subsubsection{Mars orientation parameters according to $I A U$}

IAU also defines the orientation parameters for the different planets of the Solar System (Archinal et al., 2011). The angles used by IAU to define Mars orientation are represented on Figure 2 .

Those parameters are defined with respect to the ICRF equator, a reference frame very close to the dynamical J2000 reference frame (with a precision of about 0.1 arcsecond, corresponding to about $94 \mathrm{~m}$ on Mars surface). The true North direction is defined by right ascension $\alpha_{o}$ and declination $\delta_{o}$, and the prime meridian defined by the angle $W$ with respect to the Martian equator node. The numerical values given by IAU for Mars are

$$
\begin{aligned}
\alpha_{o} & =317.68143-0.1061 T \\
\delta_{o} & =52.88650-0.0609 T \\
W & =176.630+350.89198226 d,
\end{aligned}
$$




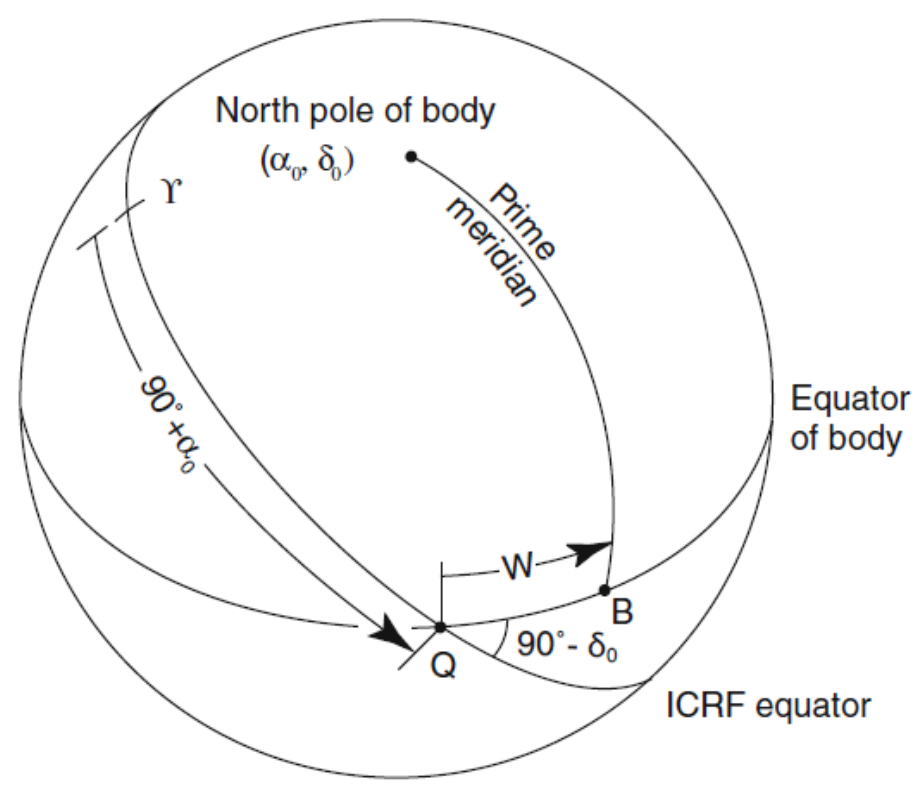

Figure 2: Mars orientation parameters as defined by IAU

where $T$ is time since J2000 expressed in centuries and $d$ time since J2000 expressed in terrestrial days. This orientation model for Mars does not include the nutation and is also based on older observations than the model given by Konopliv et al. (2016). The error induced on the true North direction when recent observations and nutation are not included in the orientation parameters calculation could then be quantified by comparing the two different orientation models.

\subsection{Planetocentric/planetographic coordinates}

The planet oblateness is introduced by a transformation from planetocentric coordinates to planetographic coordinates. The planetographic coordinates are based on the surface normal, while the planetocentric coordinates are based on the planet center. The transformation from planetographic latitude $\phi$ to planetocentric latitude $\phi^{\prime}$ is given by (e.g. Meeus (1998))

$$
\tan \phi^{\prime}=(1-f)^{2} \tan \phi,
$$

where $f$ is the oblateness coefficient or flattening ${ }^{2}$ defined as $\frac{b}{a}=1-f$ with $a$ and $b$ being respectively the planet equatorial and polar radius. On Mars, at $\phi^{\prime}=45^{\circ}$, we obtain $\phi-\phi^{\prime}=$ $0.3893^{\circ}$, i.e. a maximum difference of about 23 kilometers on the surface. However, close to the equator ( $\phi$ close to 0$)$, the difference between $\phi$ and $\phi^{\prime}$ is small.

\subsection{Transformation to the local Martian reference frame}

The Sun right ascension $\alpha_{o}$ and declination $\delta_{o}$ in the Martian equatorial reference frame can be calculated from the Sun position vector $\vec{r}_{s}$ expressed in the areocentric reference frame $(O, X, Y, Z)$.

\footnotetext{
${ }^{2}$ The value of $f$ used here is $f=0.006772$ as given by Urban and Seidelmann (2014)
} 
Thus, for a Martian observer, the hour angle $H$ is given by

$$
H=T_{s}-\alpha=\Phi+L-\alpha,
$$

where $T_{s}$ is the sidereal time, $\Phi$ is the prime meridian longitude and $L$ is the observer longitude measured with respect to the prime meridian (Fig. 3).

Here, $\alpha$ and $\delta$ coordinates are $\alpha_{o}$ and $\delta_{o}$ corrected from the parallax due to the observer location with respect to the planet center. Those corrections are said topocentric and are given by (e.g. Meeus (1998)):

$$
\begin{aligned}
\Delta \alpha & =\frac{-\Pi\left(\rho \cos \phi^{\prime} \sin H\right)}{\cos \delta}, \\
\Delta \delta & =-\Pi\left(\rho \sin \phi^{\prime} \cos \delta-\rho \cos \phi^{\prime} \cos H \sin \delta\right),
\end{aligned}
$$

where $\Pi$ is the equatorial horizontal parallax of the Sun (on Mars, $\Pi$ is approximately $3^{\prime \prime}$ ), $\rho$ is the areocentric radius taking into account the planet geometrical oblateness and $\phi^{\prime}$ is the observer planetocentric latitude.

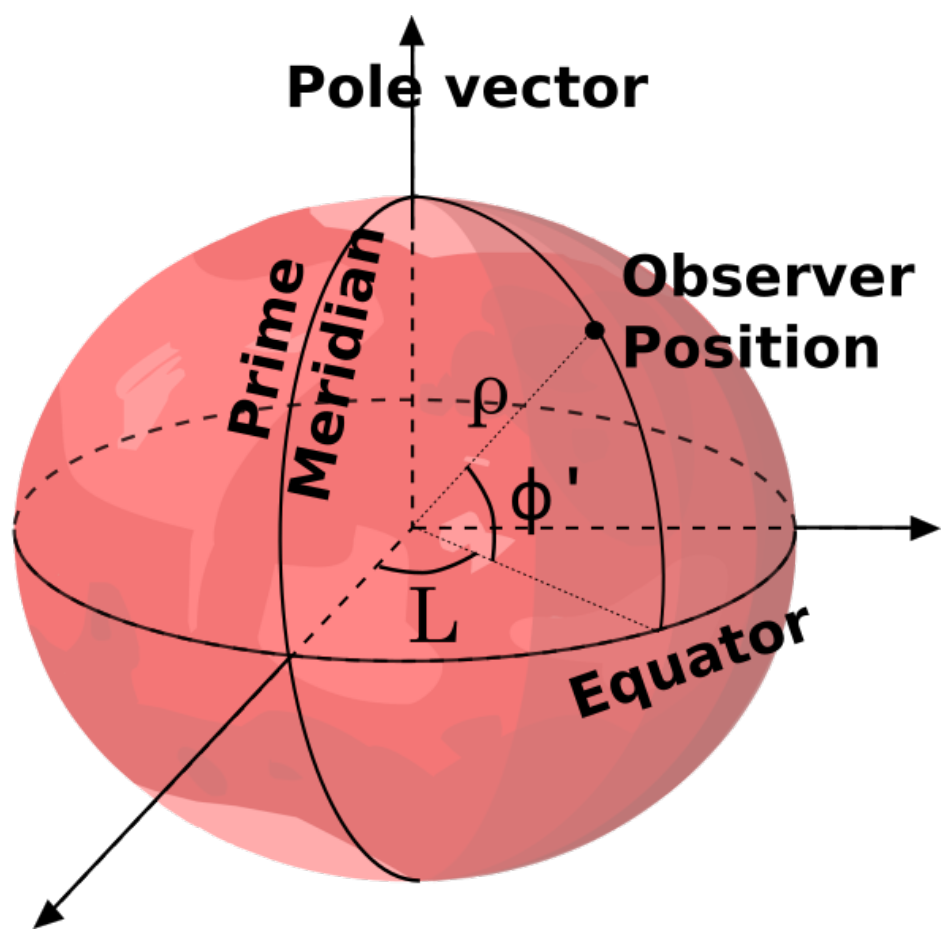

Figure 3: Angles used to determine the observer location on Mars surface.

The Sun elevation $h$ and azimuth $A$ in the Martian local reference frame are defined by

$$
\begin{aligned}
\sin h & =\sin \delta \sin \phi+\cos \delta \cos \phi \cos H, \\
\tan A & =\frac{\sin H}{\sin \phi \cos H-\cos \phi \tan \delta} .
\end{aligned}
$$

Here, $\phi$ is the planetographic latitude. The azimuth $A$ has the same sign as $H$ and is measured in the clockwise direction from true South. In the rest of the paper, the longitude and latitude given for the spacecraft position are planetographic coordinates. 
When the Sun local coordinates are known for a Martian observer, the problem can be reversed to obtain the planet orientation. If the InSight lander areographic coordinates are given, measuring the gnomon shadow position leads directly to the Sun position in the Martian sky, and by extension to the true North direction. All the concepts and calculations required to solve this problem have been detailed for the Earth by Savoie (2007) (pages 63-81) .

\section{Computing the sun position on Mars}

\subsection{Reference for local solar time}

The actual reference to determine the local solar time on Mars is an algorithm developped by Allison and McEwen (2000). This algorithm is used in the application Mars $24^{3}$ and as reference for the timing of different space missions on Mars. We computed this algorithm in order to validate our results using VSOP87 and the different orientation parameters given by Konopliv et al. (2016) or Archinal et al. (2011).

In this model, the definition of the Sun declination is given by (Allison, 1997)

$$
\delta_{S}=\arcsin 0.42565 \sin L_{s}+0.25^{\circ} \sin L_{s},
$$

where $L_{s}$ is the areocentric solar longitude.

The last term of this equation is introduced to express the change from planetocentric to planetographic coordinates. Introducing this planetographic correction to the declination thus induces a difference of maximum $0.25^{\circ}$ for $L_{s}=90^{\circ}$ or $L_{s}=270^{\circ}$, corresponding to the epochs of solstices. We also point out the fact that if $L_{s}=0^{\circ}$ or $L_{s}=180^{\circ}$ (corresponding to the epochs of equinoxes), then the corrected declination $\delta_{S}$ expressed above is not affected by any planetographic correction. This particularity is not satisfactory because the declination is used to determine the Sun local coordinates (see equations (11) and (12)). Thus it should still be affected by the planetary oblateness and observer location no matter the Sun longitude value.

In this study, Mars flattening is introduced by corrections in the observer latitude (equation 7). Those corrections are maximum for $\phi^{\prime}=45^{\circ}$ and null for $\phi^{\prime}=0^{\circ}$. But the declination is also corrected from parallax induced by the observer location on Mars. This parallax depend on the observer latitude, making the Sun declination, and thus local coordinates, latitude-dependent parameters. This dependency is not found in Allison's algorithm.

\subsection{Results}

We computed the Sun coordinates by using the Konopliv orientation model (labelled KON in the following), the IAU orientation model (labelled IAU in the following) and Allison's algorithm (labelled ALL in the following) for a mean expected InSight landing site $\left(137^{\circ} \mathrm{E}, 4^{\circ} \mathrm{N}\right)$. For the date of December 13 2018, the differences between KON and ALL for the different angles are plotted in Figure 4 . The differences between the Sun local coordinates obtained using KON and IAU are plotted on Figure 5 while the differences between ALL and IAU are plotted on Figure 5.

\footnotetext{
${ }^{3}$ https://www.giss.nasa.gov/tools/mars24/
} 


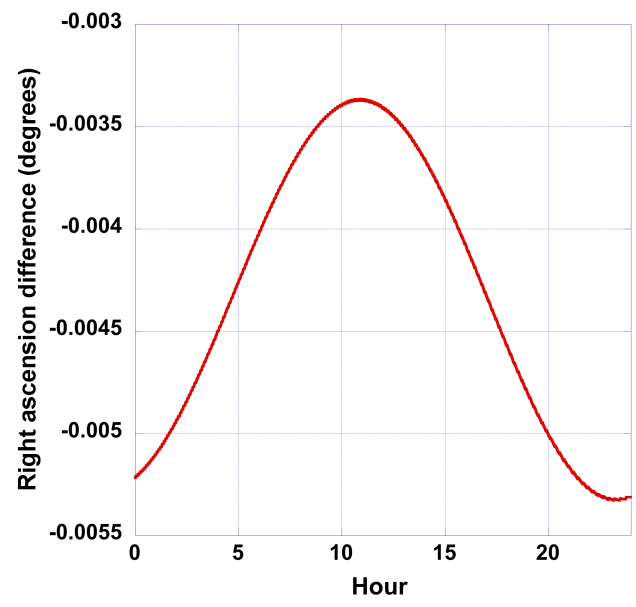

(a) Right ascension

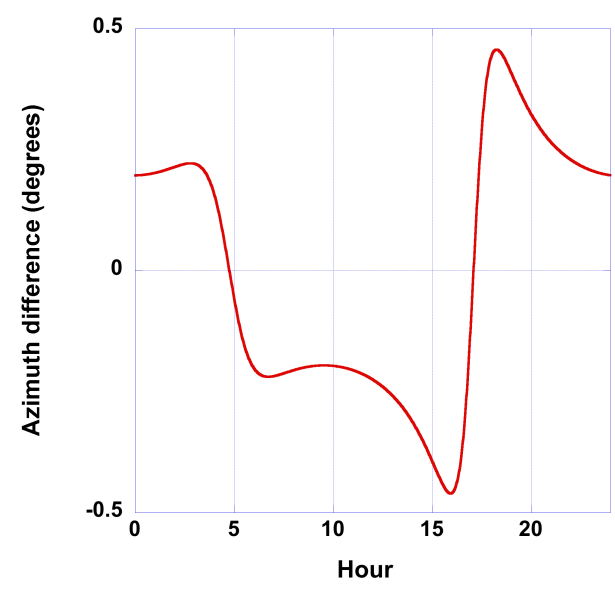

(c) Azimuth

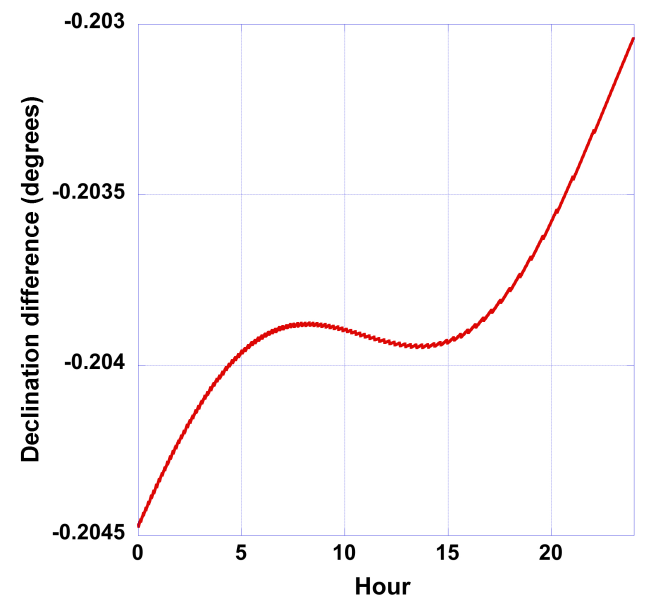

(b) Declination

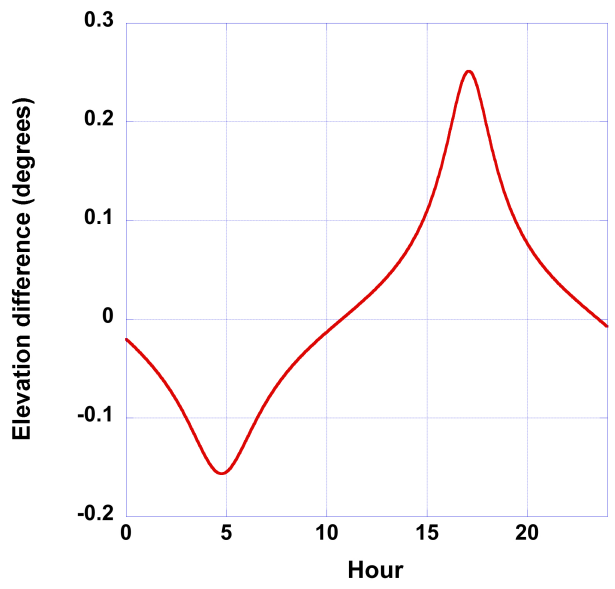

(d) Altitude

Figure 4: Comparison of the different angles for December 132018 between KON and ALL $\left(137^{\circ} \mathrm{E}, 4^{\circ} \mathrm{N}\right)$.

\subsection{Discussion}

The difference in right ascension between the KON and ALL models (Fig. 4) is below $0.005^{\circ}$, while the difference in declination is much more important, reaching an amplitude of $0.2^{\circ}$. Such a difference can be explained by the definition of the declination used in the Allison's algorithm. At the date of December $132018, L_{s}$ has a value of about $305^{\circ}$, corresponding to the $0.20^{\circ}$ difference encountered by comparing declination value with KON, corresponding to a difference of about 12 kilometers on the surface. However, we find a satisfying agreement for the equatorial coordinates by removing this planetographic correction in $\delta_{S}$.

The Sun azimuth $A$ and elevation $h$ for KON and ALL also undergo a maximum difference of $0.5^{\circ}$ and $0.25^{\circ}$ respectively. Again, those differences are located in the definition of the declination $\delta_{S}$. By changing the definition of declination in equations (11) and (12), both azimuth and elevation are impacted. However, this difference between KON and ALL in $A$ or $h$ would certainly not be 


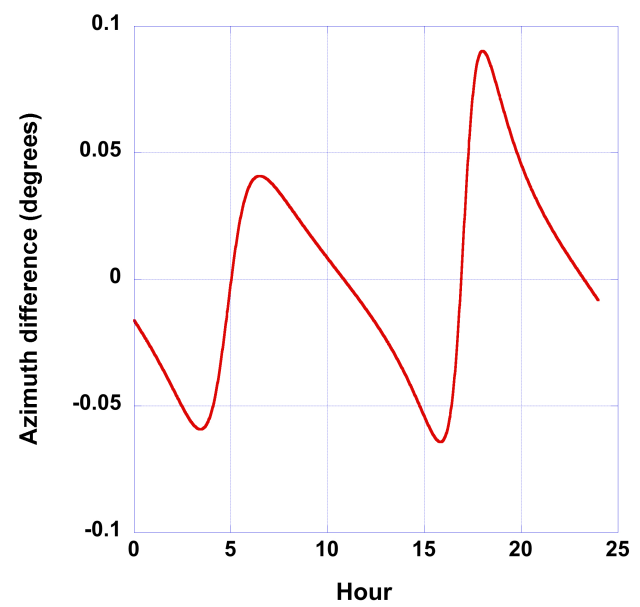

(a) Azimuth

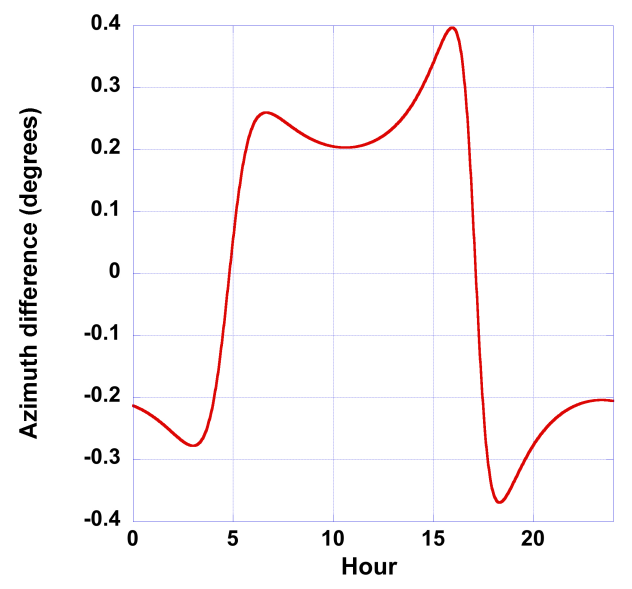

(c) Azimuth

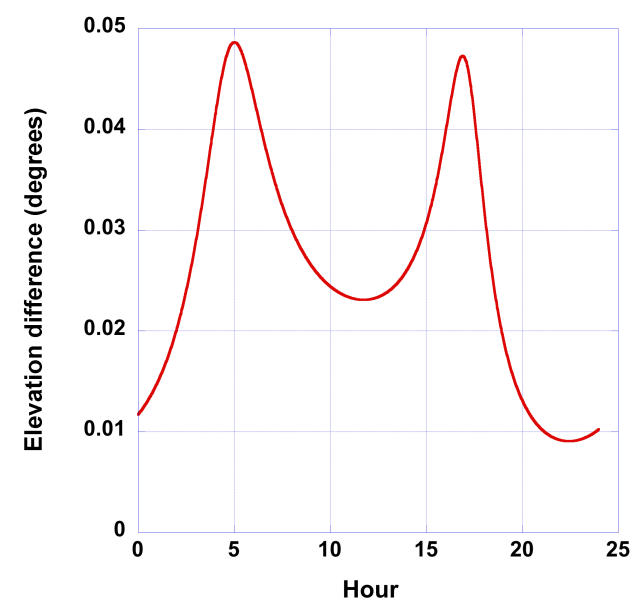

(b) Elevation

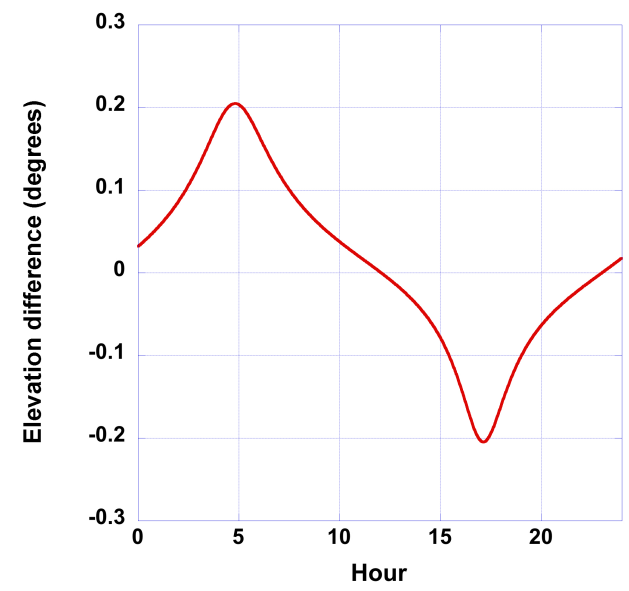

(d) Elevation

Figure 5: (a) and (b): Difference of coordinates for December 132018 between KON and IAU $\left(137^{\circ} \mathrm{E}, 4^{\circ} \mathrm{N}\right)$. (c) and (d): Difference of coordinates for December 132018 between ALL and IAU $\left(137^{\circ} \mathrm{E}, 4^{\circ} \mathrm{N}\right)$.

measurable considering the accuracy of the actual SEIS gnomon experiment.

By comparing the IAU and KON orientation models (Fig. 5, (a) and (b)), we determine a difference below $0.1^{\circ}$ in azimuth and below $0.05^{\circ}$ in elevation. These differences between the two orientation models would not be detected on the gnomon images.

Differences between ALL and IAU models (Fig. 5, (c) and (d)) are below 0.5 in azimuth and $0.25^{\circ}$ in elevation. The differences match those obtained between KON and ALL (Figure 4 ), and are again due to the planetographic correction introduced in $\delta_{S}$ in Allison's algorithm. Without that correction, Allison's algorithm is coherent with the IAU orientation model on both local coordinates $A$ and $h$.

Despite the slight differences found between the different orientation models, the results are in good agreement with each other. The differences induced by different orientation parameters or definition of the planetographic coordinates are below $0.5^{\circ}$ and would thus not be discernible on the gnomon experiment (see Section 4). For the rest of this paper, we use the most recent 
orientation model given by Konopliv et al. (2016).

\section{Possible errors committed on the true North direction}

Several type of errors can alterate the azimuth read on the target on SEIS seismometer. The main errors are:

1. errors due to an incorrect estimation of the shadow position on the camera images

2. errors due to an incorrect latitude of the lander.

3. errors due to an incorrect longitude or UTC value for the lander.

4. errors due to the gnomon non-verticality.

\subsection{Estimation of the shadow position}

The first error is the most important one. The error in the shadow position estimation will depend on the images quality, the angle of viewing, the weather and the Sun position in the sky. With the methods developed in this paper (see Section 4), the accuracy on the shadow position is estimated to be within $1^{\circ}$. This error corresponds to the reading of the shadow position on one single image. Multiple images during a day could reduce the final error on the true North direction below $1^{\circ}$. Nonetheless, this value is already below the required $5^{\circ}$ for the SEIS experiment orientation.

The deploying phase of the SEIS seismometer is scheduled between December 12 and December 25 2018. During that period, the Sun declination will endure a variation of about $2^{\circ}$, changing from $-20^{\circ} 24^{\prime}$ to $-18^{\circ} 15^{\prime}$. The Sun will be below the Martian equator ${ }^{4}$. No matter what the camera position is, the gnomon shadow will always move around the target in the clockwise direction.

\subsection{Impact of a lander incorrect latitude}

The InSight landing site in Elysium Planitia can be described as an ellipse extending between latitudes of $3^{\circ} \mathrm{N}$ and $5^{\circ} \mathrm{N}$ and longitudes of $134^{\circ} \mathrm{E}$ and $140^{\circ} \mathrm{E}$.

Mars equatorial radius (Urban and Seidelmann, 2014) is $3396.2 \mathrm{~km}$. Thus, by taking a mean latitude of $4^{\circ} \mathrm{N}, 1^{\prime}$ in longitude is equivalent to 985.4 meters $((59.27 / 60) \cos \phi)$ on the ground, and $1^{\prime}$ in latitude is equivalent to 987.9 meters.

The Sun azimuth $A$, measured in the clockwise direction from the true South, is given by equation (12).

If the lander is expected to be at a latitude $\phi$ but is actually located at a different latitude $\phi_{r}$, the measurement of the azimuth $A^{\prime}$ is different from the computed azimuth $A$. The error committed is $\left(A-A^{\prime}\right)$.

At the date of December 18 2018, the Sun declination will be $\delta=-19^{\circ} .51301$ at $02: 00$ UT (with $T T-U T=\Delta T=69 \mathrm{~s}$ ).

The error in azimuth (eq. 12) induced by a significant mistake of $\left(A-A^{\prime}\right)= \pm 10^{\prime}$ in latitude (corresponding to \pm 10 kilometers on the ground) is plotted on Figure 6. For an exact latitude of $\phi_{r}=4^{\circ} 10^{\prime}$ (black line), the error increases from sunrise (at $6: 00, A=-70^{\circ}$ ) and reaches a maximum $\left( \pm 11^{\prime}\right)$ about 1 h30 before the sun passes the meridian (where the error is expectedly null). Then the error increases again to reach a new maximum about 1 h30 after the meridian

\footnotetext{
${ }^{4}$ The Sun cross the Martian equator on March 232019.
} 


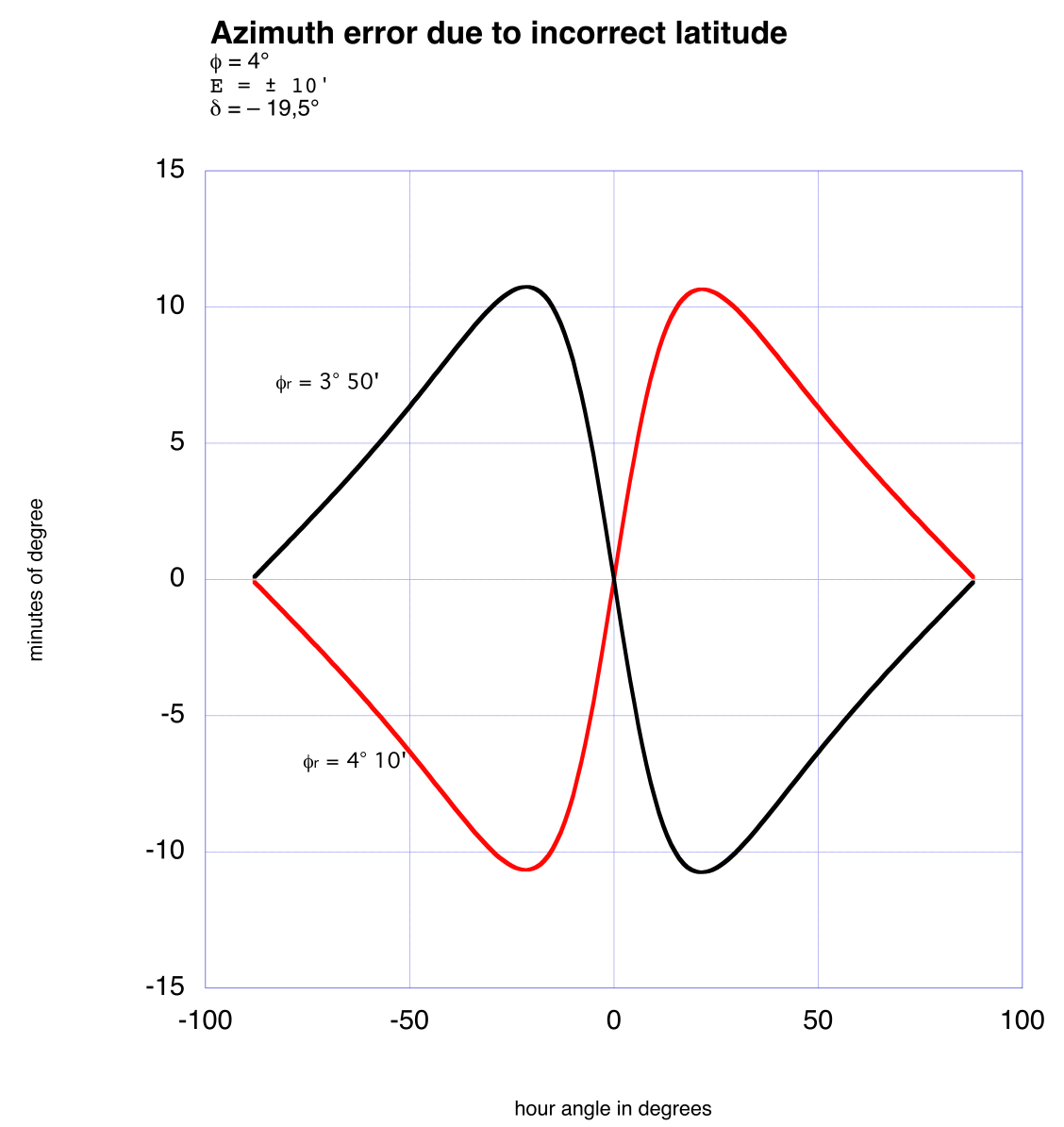

Figure 6: Evolution of the azimuth error due to a wrong value of the latitude.

crossing (the sign is changed). The error decreases afterwards until sunset (at $18: 00, A=70^{\circ}$ ). On Figure 6, the error induced by an inferior real latitude $\phi_{r}=3^{\circ} 50^{\prime}$ is also plotted (red line). The plot is symmetric to the previous one (details on the azimuth functions are given by Savoie (2007), (pages 469-480)).

To conclude, the error due to an incorrect latitude is maximum in absolute value about 1.5 hour after local solar noon. Its amplitude is noticeably identical to the shift in latitude.

\subsection{Impact of a lander incorrect longitude or incorrect UTC value}

A mistake in the InSight Martian longitude is similar to a mistake in the hour angle $H$ of the Sun; we would be calculating the azimuth for a theoretical $H$ instead of the real $H^{\prime}$. Suppose the longitude error is of $\pm 10^{\prime}$, i.e. about 10 kilometers on the ground (at December 18 2018). This error in longitude induces a shift of 40 seconds in time. The induced error in azimuth for a wrong longitude is plotted on Figure 7. The error increases between sunrise and the moment the Sun passes the meridian, with a value reaching almost a quarter of degree in absolute value $\left(0^{\circ} 24^{\prime}\right)$. In proportion, the error in longitude has a much bigger impact than the error in latitude. Also, a mistake on the UTC signal leads to an error in the local time and thus the longitude. For an error 
of 10 seconds in UTC, the Sun azimuth is wronged by $\pm 0^{\circ} 6^{\prime}$.

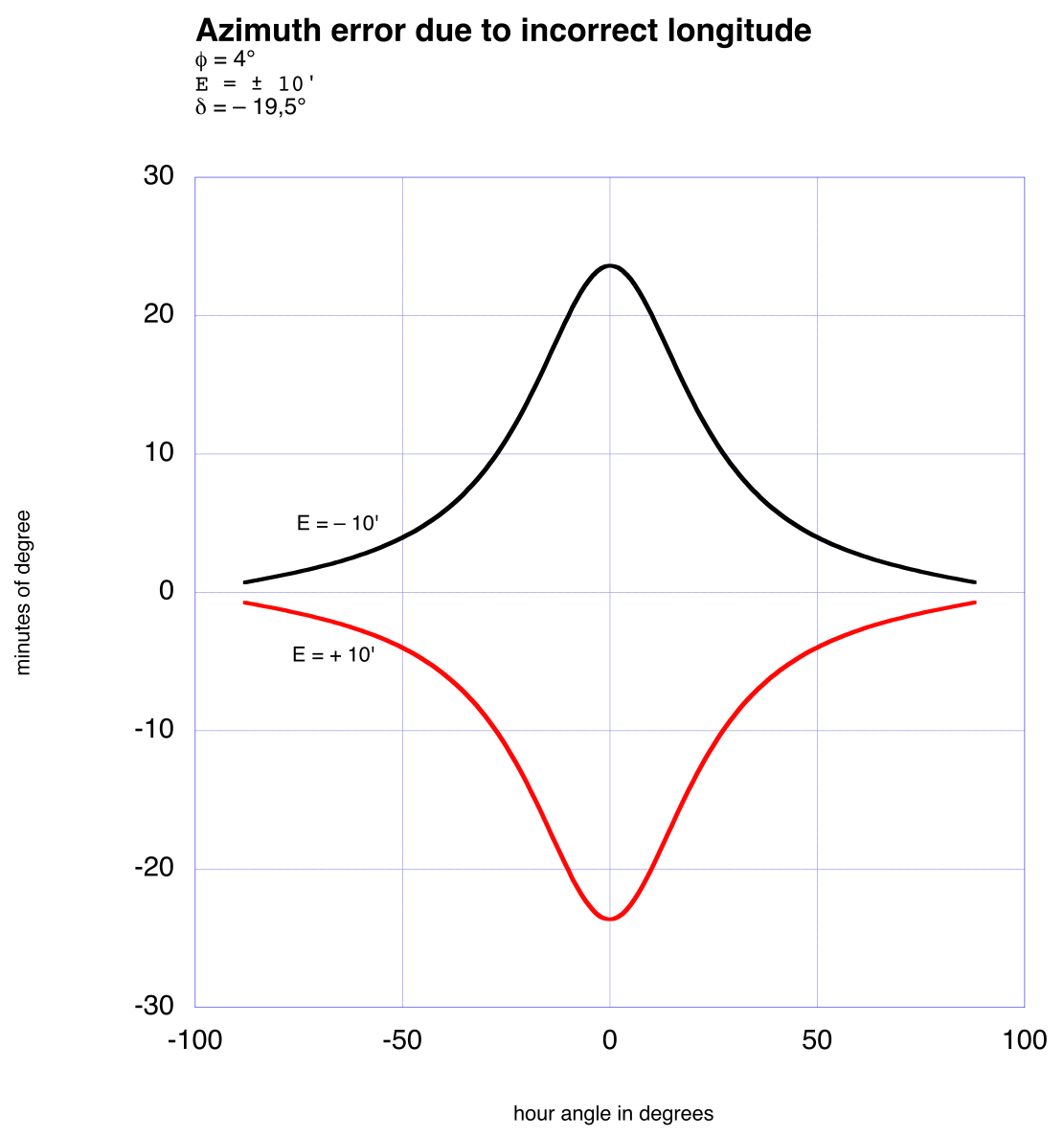

Figure 7: Evolution of the azimuth error due to a mistake in longitude.

By combining mistakes in latitude and longitude $\left(+10^{\prime}\right.$ in latitude, $+10^{\prime}$ in longitude), the total error commited in the Sun azimuth is plotted on Figure 8. We could also provide results for different situations $\left( \pm 10^{\prime}\right.$ in latitude and $\pm 10^{\prime}$ in longitude), but the conclusions would be the same: the maximum error obtained reaches $0.5^{\circ}$ about 30 minutes before or after the Sun passes the meridian.

In previous missions, the lander coordinates are known up to $4^{\prime \prime}$ in latitude (e.g. Folkner et al. (1997)). These uncertainties would be well below the reading errors on the target images.

\subsection{Consequences of the gnomon non-verticality on the Sun azimuth}

If the lander target plane is deviated from horizontality, the angle read on the target would not be the azimuth $A$ but a new angle $A^{\prime}$ (Fig. 9). Let $D$ be the gnomonic declination ${ }^{5}$ of this

\footnotetext{
${ }^{5}$ The gnomonic declination is the azimuth of the normal to the plane, giving the orientation of the plane in space
} 


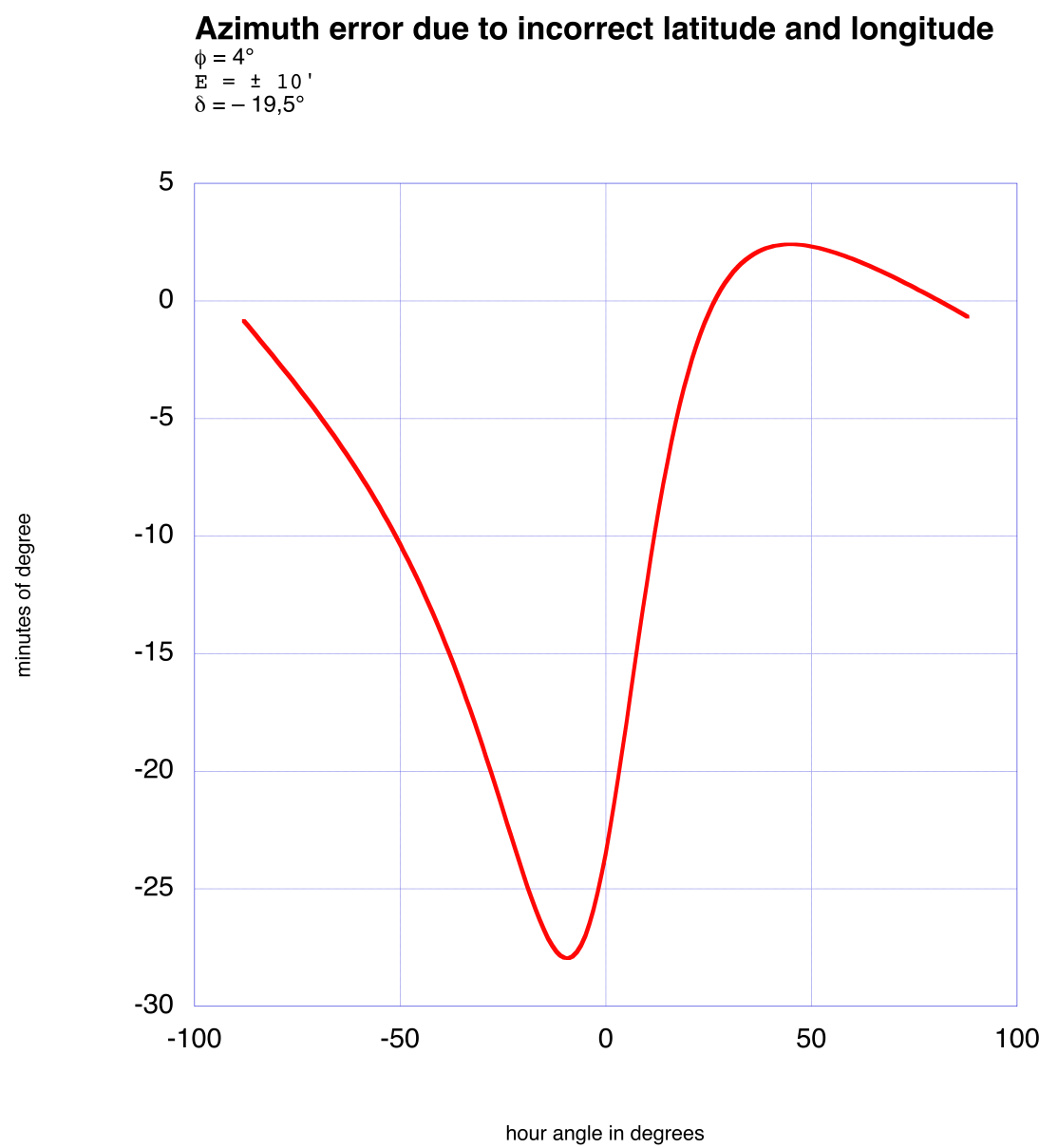

Figure 8: Evolution of the azimuth error due to the cumulation of mistakes in latitude and longitude.

inclined plane, and $z$ the gnomon inclination ${ }^{6}$. The altered Sun azimuth $A^{\prime}$ can be calculated (see Savoie (2007), (pages 103-107)) by using:

$Q=(\sin D \sin z) \sin H+\cos H(\cos \phi \cos z+\sin \phi \sin z \cos D)+\tan \delta(\sin \phi \cos z-\cos \phi \sin z \cos D)$, $N_{x}=\cos D \sin H-\sin D(\sin \phi \cos H-\cos \phi \tan \delta)$, $N_{y}=(\sin D \cos z) \sin H-\cos H(\cos \phi \sin z-\sin \phi \cos z \cos D)-\tan \delta(\sin \phi \sin z+\cos \phi \cos z \cos D)$, with

$$
\begin{aligned}
& x=\frac{N_{x}}{Q}, \\
& y=\frac{N_{y}}{Q} .
\end{aligned}
$$

\footnotetext{
${ }^{6}$ The zenithal distance from the normal to the plane.
} 


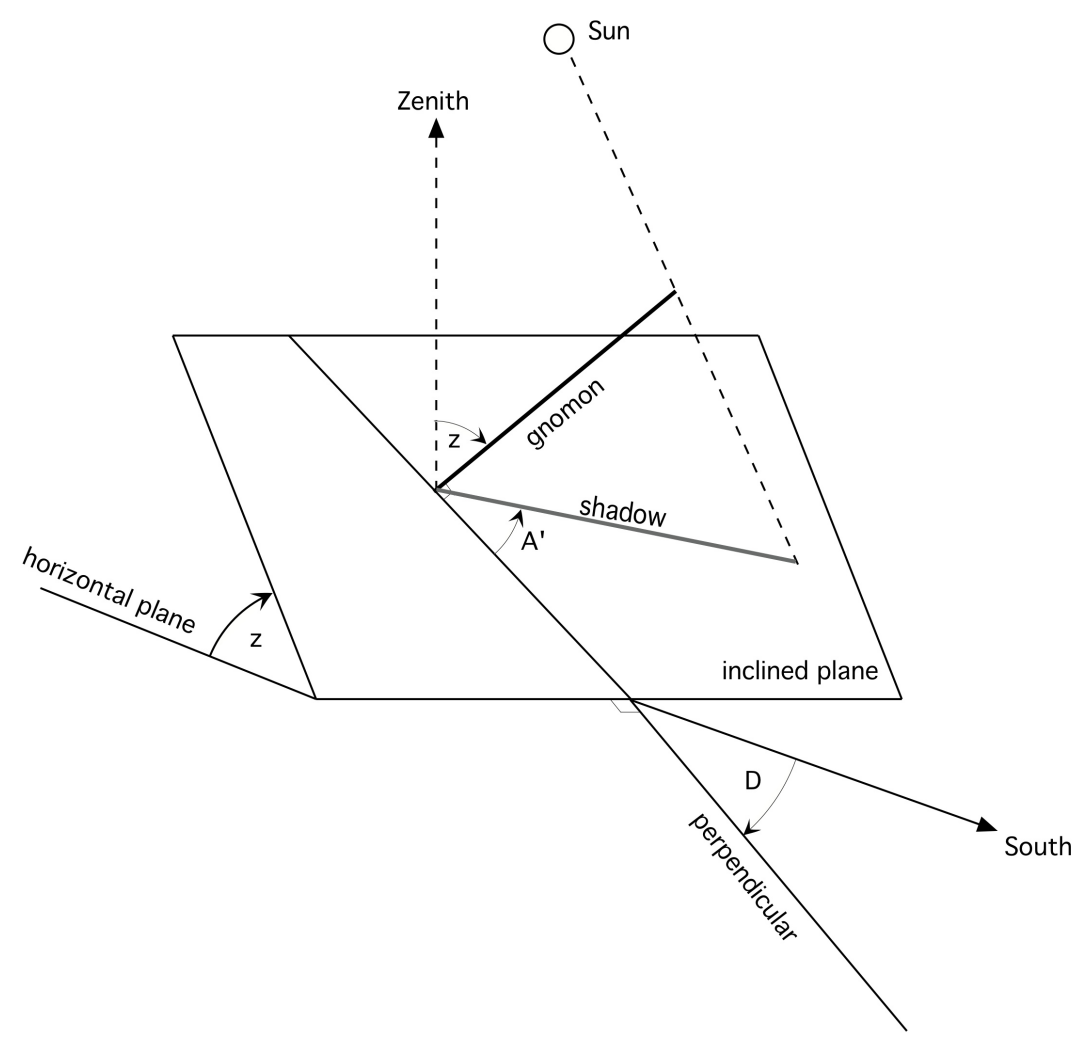

Figure 9: Target plane inclination.

Finally,

$$
\tan A^{\prime}=\frac{x \cos \Psi+y \sin \Psi}{y \cos \Psi-x \sin \Psi},
$$

with

$$
\tan \Psi=\tan D \cos z
$$

$\Psi$ being the angle between the South meridian and the line of maximum slope of the plane.

$A$ can be compared to $A^{\prime}$ by taking $z=0.1^{\circ}$ as a maximum deviation (the expected horizontality for the SEIS experiment (Lognonné et al., 2018)). The results are plotted on Figure 10 for a mean Sun declination of $\delta=-18.5^{\circ}$ and different azimuth from $0^{\circ}$ to $180^{\circ}$ every $45^{\circ}$ (South, SouthWest, West, North-West, values for East are symmetrical). The maximum error is reached near the meridian $\left( \pm 0.25^{\circ}\right)$ when the target plane face exactly West or East. In the other cases, the azimuth error stays below $\pm 5^{\prime}$ if measurements are done before $8: 40$ or after $15: 20$ (in Local Solar Time LST).

\subsection{Conclusions}

To reach a precision below $1^{\circ}$ on the Sun azimuth, and thus the true North direction, the observations of SEIS gnomon shadow should take place early after sunrise until about $8: 30$ LST, then from 15: 30 LST to the sunset.

Since the expected accuracy on the true North direction is of $5^{\circ}$, the observation window can be even larger, by still avoiding a period of 1.5 hour before and after the Sun passes the meridian. 


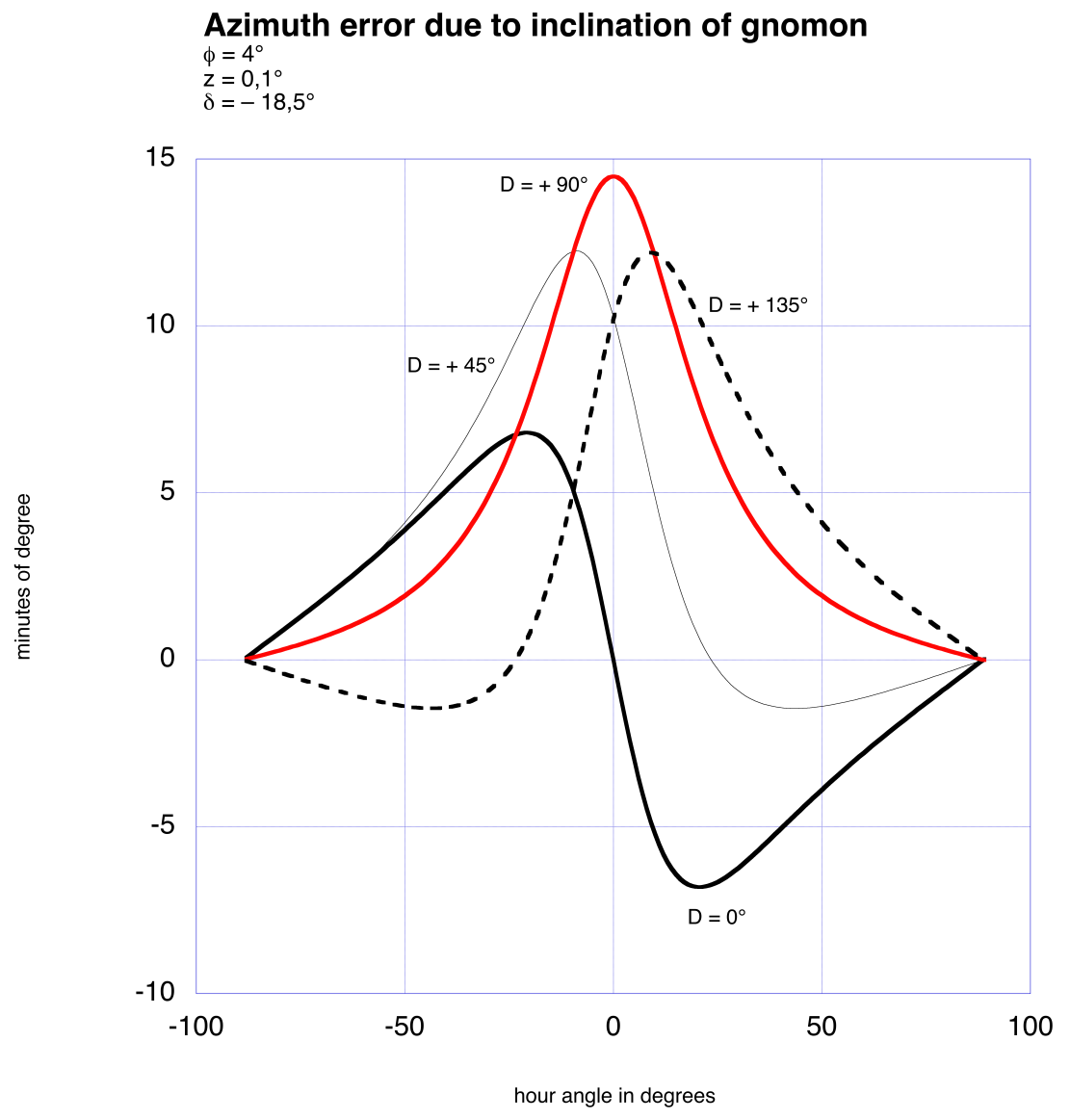

Figure 10: Evolution of the error in azimuth due to the gnomon non-verticality.

The azimuth function defined by equation (12) shows that the higher the Sun elevation is, the larger the Sun azimuth variations as a function of the hour angle are. In other words, the gnomon shadow moves faster around the meridian, making it a disadvantage. At a latitude of $4^{\circ} \mathrm{N}$, the Sun meridian elevation is about $65^{\circ}$ to $67^{\circ}$ above the southern horizon between December 12 and December 25 2018, and an apparent diameter of about 22' (inducing a penumbra less important than on the Earth).

Since the total error is increasing with days (the Sun getting closer to the equator, the declination will affect the result), it is recommended to observe the gnomon shadow as soon as deploying phase allows it: it is preferable to observe the shadow around December 13-14 than December 24-25. This time period would allow to avoid the moment when errors on longitude and latitude would induce the biggest anomalies in azimuth. Also, the shadow would be longer and thus easier to identify on the target.

The length of the gnomon shadow is computed in Table 2 (Appendix) from sunrise to sunset of December 192018 for a latitude of $4.5^{\circ} \mathrm{N}$. The hour angle $H$, Sun azimuth $A$, Sun elevation $h$ and gnomon shadow length $l$ are given in the table. Also, the Sun azimuth can only be measured when the shadow length is lower than the outer radius of the target used for measuring the shadow 
position. Thus, shadow length larger than $65 \mathrm{~mm}$ are excluded. If the time period corresponding to greater sensibility to errors (cf. supra) are avoided, the best time periods for measurement are identified (in bold), for a total time of approximately 5 hours.

However, an observation of the shadow being made when the Sun passes the meridian could be used to check the true North direction. At this exact moment, the Sun culminates in the South direction, and the gnomon shadow can confirm the post-meridian observations.

\section{True North direction}

The determination of true North on Mars is based on the observation of a gnomon shadow made by a camera mounted on the lander articulated arm (Maki et al., 2018). The exact cardinal position can be deduced by computing the Sun azimuth from the shadow position. That objective is however difficult due to the SEIS gnomon shape: there is an ellipsoidal part on top of the gnomon stick which produces a large shadow on the target. The main error we can expect in true North determination lies in the error committed in the shadow position measurement. This error measurement results from a large shadow on the target coupled with low resolution pictures.

\subsection{SEIS gnomon characteristics}

The deploying phase of SEIS seismometer is the perfect occasion to set up the very first Martian gnomon. The gnomon is a useful tool to determine, with a relative precision, the North-South axis direction. The benefit of this technic is to allow the determination of SEIS orientation by a "simple" observation of the Sun trajectory in the Martian sky, which means without any use of a satellite navigation system or gyroscopes.

The target used to measure the shadow direction and length is divided into three concentric rings (Fig. 11) (see Lognonné et al. (2018)). Those rings are segmented into dark and light zones, each one having a width of $5^{\circ}$. The zones are used to measure approximately the azimuth of the gnomon shadow. Each ring has a similar segmentation, shifted of $2.5^{\circ}$ from the adjacent rings. The inner diameter of the rings are respectively $56 \mathrm{~mm}, 80.6 \mathrm{~mm}$ and $105.4 \mathrm{~mm}$. The last ring has an outer diameter of $130 \mathrm{~mm}$.

The gnomon is located in the center of the target and made of a $16.84 \mathrm{~mm}$ long stick, covered by a hemispherical part of $10.49 \mathrm{~mm}$ (Fig. 12). The whole gnomon is also lifted, located on top of a platform $1.37 \mathrm{~mm}$ thick. The gnomon has a total height of $28.7 \mathrm{~mm}$. The top of the shadow projected by this hemispherical part is the point used to determine the Sun position in the sky. The difficulty lies in the precise reading of the shadow top position, as confirmed by the preliminary images on Figure 11.

\subsection{Images of the sundial}

Images of the sundial are acquired by the Instrument Deployment Camera (IDC), described in Maki et al. (2018). The IDC has an angular resolution of approximately 0.82 milli-radians/pixel at the center of the image and a field of view of 45 degrees (horizontal) x 45 degrees (vertical). During InSight surface operations, IDC images of the sundial will be acquired with the camera located approximately 1 meter above the sundial, with the sun illuminating the sundial approximately from the south and the camera located approximately to the north of the sundial. The exact details of the camera geometry depend on the actual placement of the SEIS on Mars with respect to the lander, but generally speaking the geometry will be similar to the configuration shown in Figure 


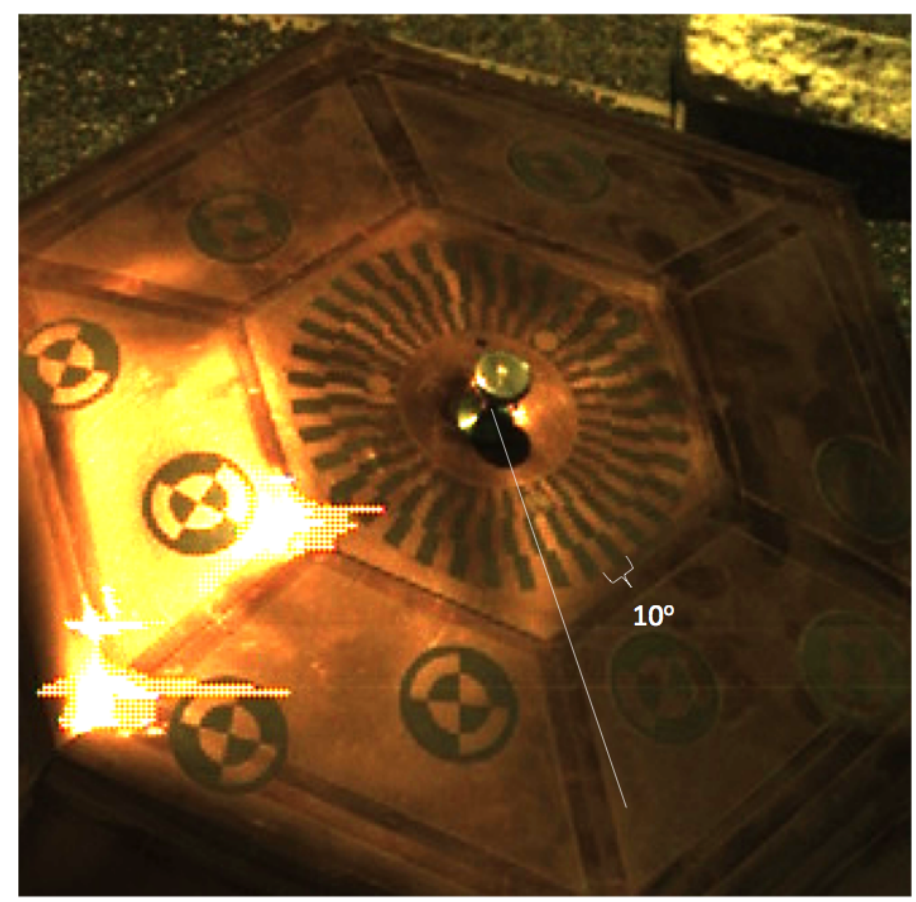

Figure 11: Picture of the gnomon and target above the SEIS seismometer.

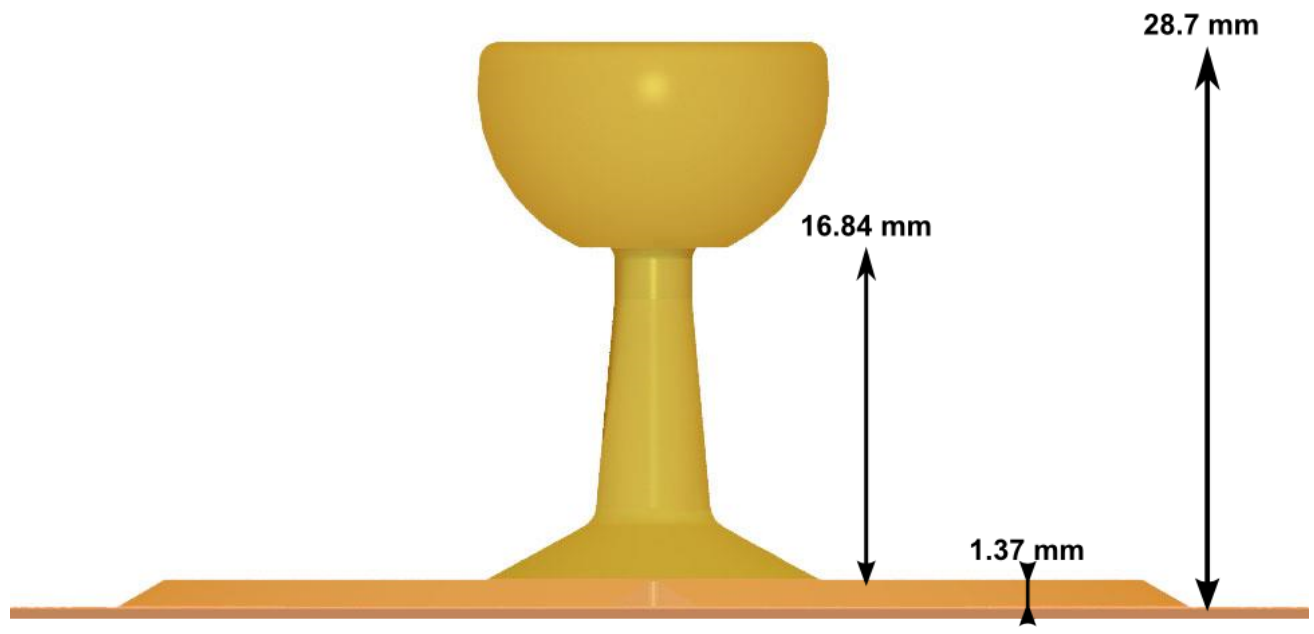

Figure 12: Schematic of the gnomon used on SEIS.

13. The sundial images will be JPEG-compressed prior to transmission, at JPEG compression quality values of approximately 85 (Maki et al., 2018). Once received on Earth, the images will be used to measure the location of the gnomon shadow. Three images will be specifically dedicated to sundial imaging: one at noon Local True Solar Time (LTST), and one in the morning and evening. The images will be time tagged with acquisition times to enable lookup of solar ephemeris data. 


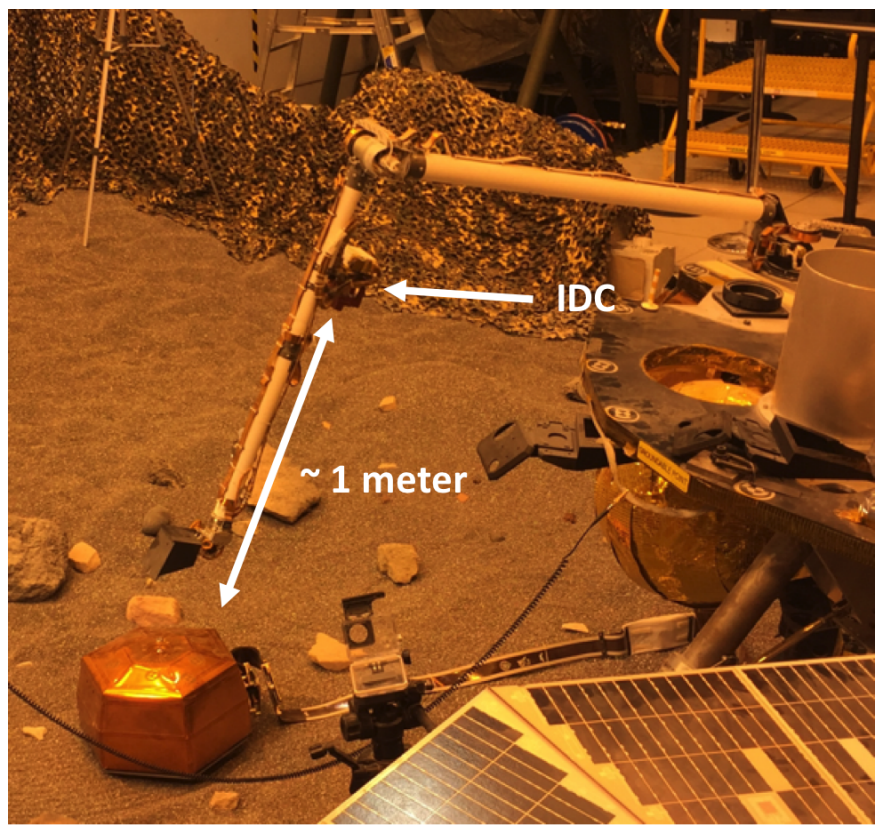

Figure 13: SEIS sundial image acquisition by the Instrument Deployment Camera in the InSight testbed at the Jet Propulsion Laboratory in Pasadena, CA.

\subsection{Simulated observations}

We generated simulated images of the gnomon and target by using the open-source software POV-Ray ${ }^{7}$. POV-Ray is a software of "ray tracing", enabling to simulate the gnomon and its shadow on the target. We included the Sun theory developped in the first part of this paper and took into account the apparent size of the Sun and thus the penumbra induced.

It is then easy to introduce some errors in longitude, latitude or even non-horizontality of the target. Those errors can be introduced randomly in the simulation with the values saved in a separate file. The images simulated are then deteriorated to get a final render as close as possible to the real images expected with the InSight camera (Fig. 14).

We can then work in an almost real situation to define the methodology to measure the shadow position and estimate the expected precision in the Sun azimuth and elevation, and thus in the true North direction.

\subsection{Methods}

As seen from the simulated images on Figure 14, the exact location of the shadow top is difficult to determine. To optimise the estimation of the shadow position, different options are possible.

\subsubsection{Shadow on the rings borders}

The exact moment when a contact occurs between the shadow top and the target rings borders can be determined. The calculation of those events takes into account the odd shape of the gnomon, especially the rounded edges of the top. Thus, the borders of the rings on the target can be used to complement the Sun azimuth as an indicator to the Sun elevation. By using a gnomon of 28.7

\footnotetext{
${ }^{7}$ www.povray.org
} 

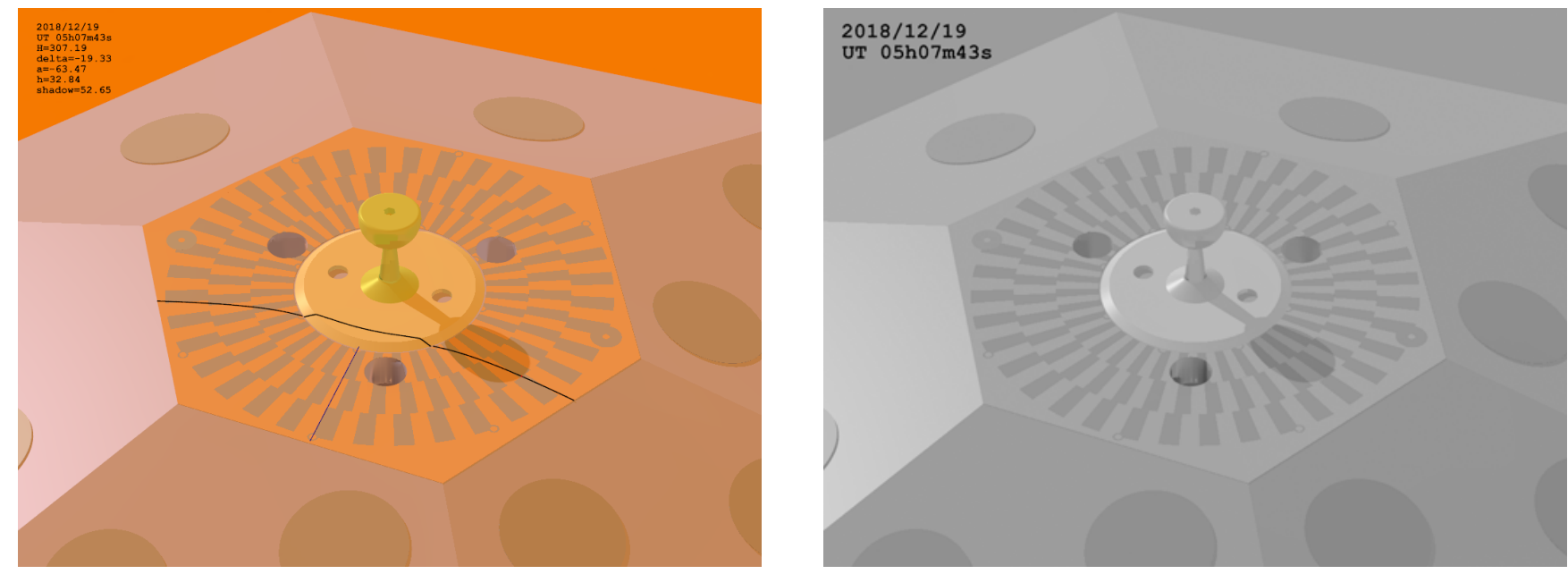

Figure 14: Gnomon images simulated with the open-source software POV-Ray. On the left, the image shows the simulation with the trajectory of the top of the shadow traced in black for the day of December 19 2018. The direction of true North, corresponding to the minimum shadow length, is plotted in black. On the right, the image has been deteriorated to reproduce the quality of the onboard camera and verify the validity of the method.

$\mathrm{mm}$ high, the shadow will be in contact with the rings borders when the Sun reaches an elevation $h$ such as (see Fig. 15):

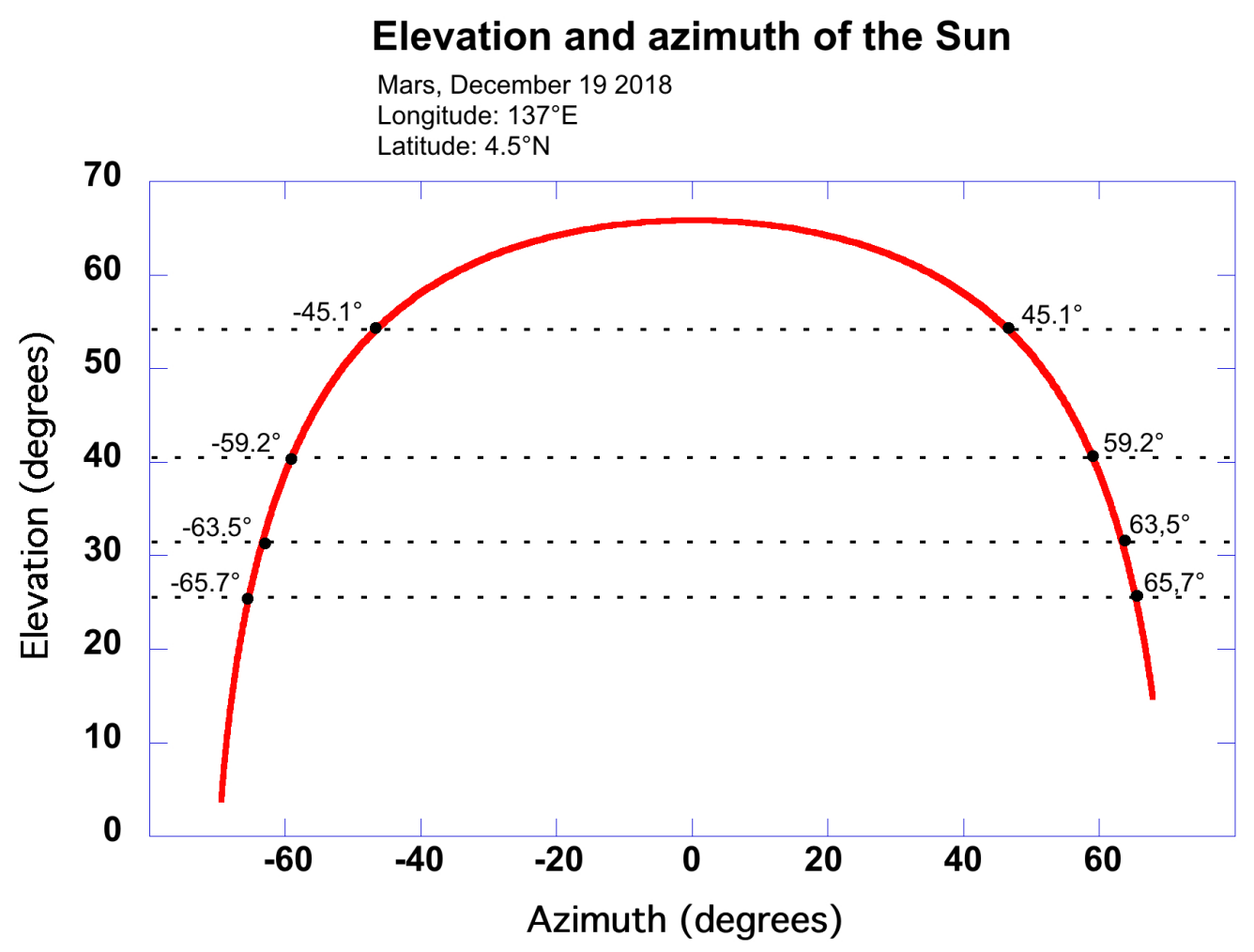

Figure 15: Sun elevation as a function of its azimuth for the InSight lander on December 19 2018. The elevations required to obtain a point of contact between the gnomon shadow and the target rings borders are traced in dotted lines, the corresponding azimuth values indicated below. 
- $h=26.77^{\circ}$ for a target radius of $65 \mathrm{~mm}$,

- $h=32.83^{\circ}$ for a target radius of $52.7 \mathrm{~mm}$,

- $h=41.83^{\circ}$ for a target radius of $40.3 \mathrm{~mm}$,

- $h=55.70^{\circ}$ for a target radius of $28 \mathrm{~mm}$.

As soon as the shadow length exceeds $65 \mathrm{~mm}$, the target cannot be used for measuring the Sun elevation. Thus, optimal time zones can be determined long before arrival on Mars for InSight's camera to take images of the SEIS sundial.

Taking pictures at the exact moment of contacts is also a way to control the validity of the UTC signal. Any error on UTC would induce a picture taken too soon or too late, and would induce a delay to the moment of contact between the shadow and the borders.

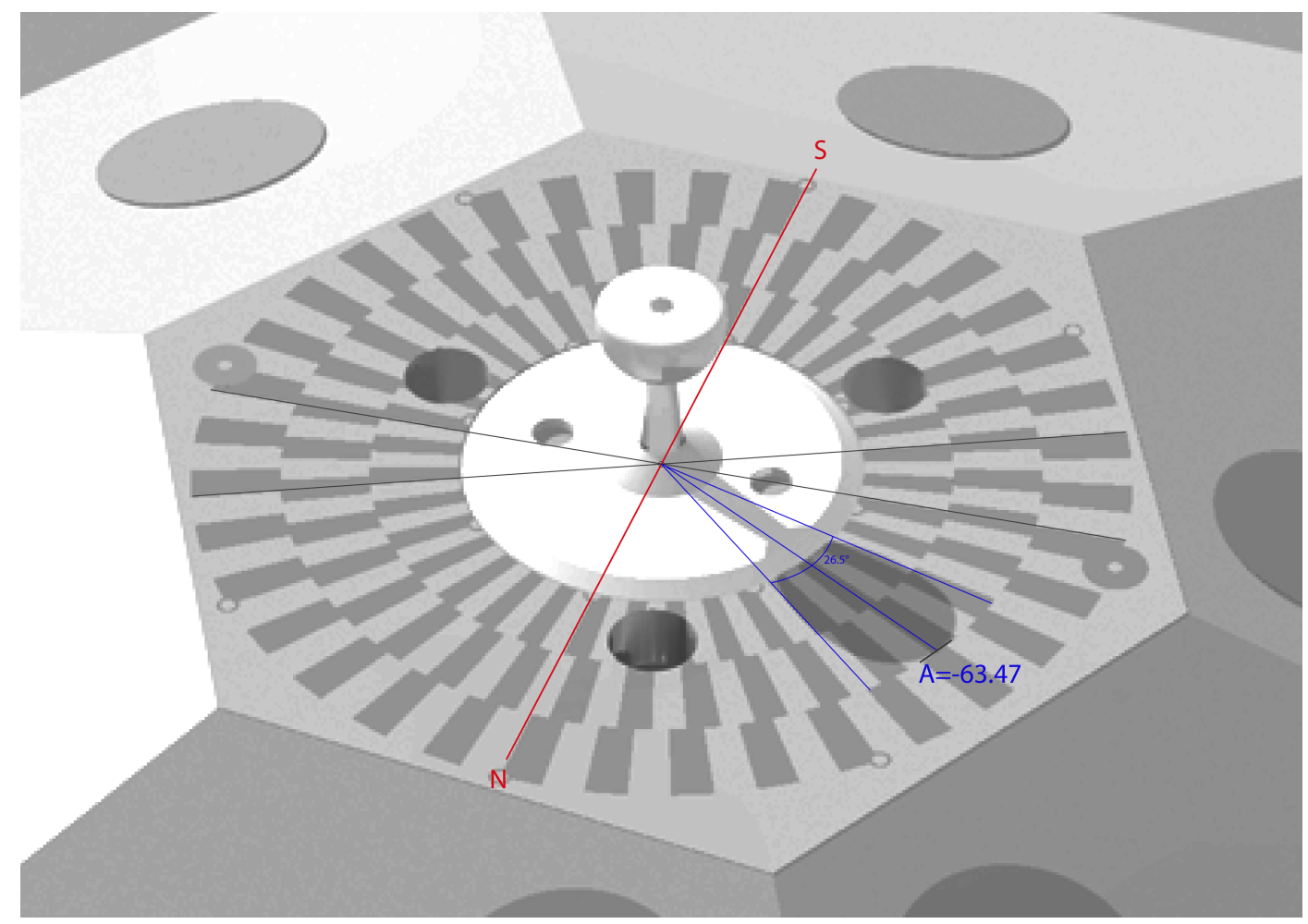

Figure 16: Simulation of the gnomon shadow on December 192018 at 05 : 07 UT.

For our example of December 19 2018, Figure 16 shows the shadow position during a contact on the outer border of the second ring. The contact occurs at 05 : 07 UT. The Sun elevation associated is $32.84^{\circ}$ in the Martian sky. The Sun ephemeris computed for this location, date and time provides the azimuth and elevation of the Sun (see Fig. 15), and enables to determine the true North-South axis direction from which it is measured.

When the Sun crosses the meridian, the shadow length is at its minimum, below $28 \mathrm{~mm}$ and out of the target. Its azimuth can not be determined on images taken at that specific time but the shadow direction can still be used to confirm the estimated true North direction by other images. 


\subsubsection{Random shadow position}

For images taken at a random time when the shadow is on the target, the shadow length is not precisely determined. In that case, the method consists in identifying the borders of the shadow, and finds the median line pointing to the shadow top. The azimuth of the Sun (and thus the shadow) is known with the ephemeris for the time of the picture, thus the North-South axis can be estimated on the image.

On Figure 16, the shadow borders are marked. The median line is plotted and provides the opposite direction of the Sun. Computing the Sun azimuth $\left(A=-63.47^{\circ}\right)$ for the corresponding location, date and time gives the true North-South axis direction.

\subsubsection{Multiple images}

If several images are taken during local mornings and evenings, the shadow trajectory can be extrapolated from the measured positions. As seen on Figure 14, the Shadow follows a path on the target that can be simulated with POV-Ray. The shortest shadow length, when the Sun crosses the meridian, can then be sorted out and provides the North-South axis direction.

\section{Conclusion}

Determining the true North direction by using the Sun is a foolproof technic on the Earth. For the first time, the InSight mission will enable to reproduce on Mars one of the most ancient astronomical observation. SEIS seismometer will use the first Martian gnomon, whose shadow can be used to determine local solar time, lander position and orientation. In order to analyse the future observation of the Martian gnomon, we computed the Sun local coordinates on Mars by using the VSOP87 planetary ephemeris.

The coordinates obtained have been compared to the actual reference used by NASA for Mars rovers local time. The algorithm developed by Allison and McEwen (2000) validates our calculations, despite a slight difference due to the chosen definition of declination also impacting the local coordinates. This difference is however negligible considering the actual precision of the Martian gnomon, and the fact that the chosen landing site is close to the Martian equator.

We explored the different possible sources of errors in the shadow position due to an incorrect lander position or to the non-verticality of the gnomon. We thus determined the optimum periods to observe the shadow and reduce the possible errors impact, a total of about 5 hours split between mornings and evenings.

The simulation of the gnomon shadow using a ray-tracing software enables to explore the best ways to determine the true North direction using only images of the shadow. Despite different possible error sources, the main part of the error on the true North direction comes from the estimation of the shadow top position on the images. With images taken by the camera at precise moments required by singular events (point of contact between the shadow and the rings borders), or randomly taken between mornings and evenings optimal periods if UTC is precisely known, the true North direction can be deduced with a precision of about $1^{\circ}$ of accuracy, well within the $5^{\circ}$ required by the SEIS experiment.

\section{References}

M. Allison. Accurate analytic representations of solar time and seasons on Mars with applications to the Pathfinder/Surveyor missions. Geophys. Res. Lett., 24:1967, August 1997. 
M. Allison and M. McEwen. A post-Pathfinder evaluation of areocentric solar coordinates with improved timing recipes for Mars seasonal/diurnal climate studies. Planet. Space Sci., 48:215235, February 2000.

B. A. Archinal, M. F. A'Hearn, E. Bowell, A. Conrad, G. J. Consolmagno, R. Courtin, T. Fukushima, D. Hestroffer, J. L. Hilton, G. A. Krasinsky, G. Neumann, J. Oberst, P. K. Seidelmann, P. Stooke, D. J. Tholen, P. C. Thomas, and I. P. Williams. Report of the IAU Working Group on Cartographic Coordinates and Rotational Elements: 2009. Celestial Mechanics and Dynamical Astronomy, 109:101-135, February 2011.

P. Bormann, K. Klinge, and S. Wendt. Data Analysis and Seismogram Interpretation. IASPEI, GFZ German Research Centre for Geosciences, 2009.

M. Böse, J. F. Clinton, S. Ceylan, F. Euchner, M. van Driel, A. Khan, D. Giardini, P. Lognonné, and W. B. Banerdt. A probabilistic framework for single-station location of seismicity on Earth and Mars. Physics of the Earth and Planetary Interiors, 262:48-65, January 2017.

C. Braak. On the determination of the epicentre of earht-quake by means of records at a single station. Koninklijke Nederlandse Akademie van Wetenschappen Proceedings Series B Physical Sciences, 13:11-16, January 1910.

P. Bretagnon. Theory for the motion of all the planets - The VSOP82 solution. A\& A, 114:278-288, October 1982.

P. Bretagnon and G. Francou. Planetary theories in rectangular and spherical variables - VSOP 87 solutions. A $G A, 202: 309-315$, August 1988.

J. Clinton et al. The Marsquake Service: securing daily analysis of SEIS data and building the Martian seismicity catalogue for InSight. Space Sci. Rev., this issue, 2018.

I. Daubar et al. Impact-Seismic Investigations of the InSight Mission. Space Sci. Rev., this issue, 2018.

W. M. Folkner, C. F. Yoder, D. N. Yuan, E. M. Standish, and R. A. Preston. Interior Structure and Seasonal Mass Redistribution of Mars from Radio Tracking of Mars Pathfinder. Science, 278:1749, December 1997.

M. Golombek, D. Kipp, N. Warner, I. J. Daubar, R. Fergason, R. L. Kirk, R. Beyer, A. Huertas, S. Piqueux, N. E. Putzig, B. A. Campbell, G. A. Morgan, C. Charalambous, W. T. Pike, K. Gwinner, F. Calef, D. Kass, M. Mischna, J. Ashley, C. Bloom, N. Wigton, T. Hare, C. Schwartz, H. Gengl, L. Redmond, M. Trautman, J. Sweeney, C. Grima, I. B. Smith, E. Sklyanskiy, M. Lisano, J. Benardini, S. Smrekar, P. Lognonné, and W. B. Banerdt. Selection of the InSight Landing Site. Space Sci. Rev., 211:5-95, October 2017.

B. Kenda, P. Lognonné, A. Spiga, T. Kawamura, S. Kedar, W. B. Banerdt, R. Lorenz, D. Banfield, and M. Golombek. Modeling of Ground Deformation and Shallow Surface Waves Generated by Martian Dust Devils and Perspectives for Near-Surface Structure Inversion. Space Sci. Rev., 211:501-524, October 2017. 
A. Khan, M. van Driel, M. Böse, D. Giardini, S. Ceylan, J. Yan, J. Clinton, F. Euchner, P. Lognonné, N. Murdoch, D. Mimoun, M. Panning, M. Knapmeyer, and W. B. Banerdt. Singlestation and single-event marsquake location and inversion for structure using synthetic Martian waveforms. Physics of the Earth and Planetary Interiors, 258:28-42, September 2016.

A. S. Konopliv, C. F. Yoder, E. M. Standish, D.-N. Yuan, and W. L. Sjogren. A global solution for the Mars static and seasonal gravity, Mars orientation, Phobos and Deimos masses, and Mars ephemeris. Icarus, 182:23-50, May 2006.

A. S. Konopliv, R. S. Park, and W. M. Folkner. An improved JPL Mars gravity field and orientation from Mars orbiter and lander tracking data. Icarus, 274:253-260, August 2016.

P. Lognonné, B. Banerdt, D. Giardini, U. Christensen, T. Pike, D. Mimoun, S. de Raucourt, S. Tillier, P. Zweifel, D. Mance, R. Roll, M. Bierwirth, L. Boschi, R. Garcia, W. Goetz, C. Johnson, N. Kobayashi, A. Mocquet, M. Panning, J. Tromp, R. Weber, and M. Wieczorek. The GEMS (GEophysical Monitoring Station) SEISmometer. In EPSC-DPS Joint Meeting 2011, page 1507, October 2011.

P. Lognonné and C.L. Johnson. Planetary Seismology. Elsevier, Oxford, 2015.

P. Lognonné et al. The SEIS: The Seismic Experiment for Internal Structure of InSight. Space Sci. Rev., this issue, 2018.

J.N. Maki, M. Golombek, R. Deen, H. Abarca, C. Sorice, T. Goodsall, M. Schwochert, M. Lemmon, A. Trebi-Ollennu, and B. Banerdt. The Color Cameras on the InSight Lander. Space Sci. Rev., submitted, 2018.

J. Meeus. Astronomical algorithms. Willmann-Bell, 1998.

M.P. Panning, E. Beucler, M. Drilleau, A. Mocquet, P. Lognonné, and W.B. Banerdt. Verifying single-station seismic approaches using Earth-based data: Preparation for data return from the InSight mission to Mars. Icarus, 248:230-242, March 2015.

D. Reiss, L. Fenton, L. Neakrase, M. Zimmerman, T. Statella, P. Whelley, A. P. Rossi, and M. Balme. Dust Devil Tracks. Space Sci. Rev., 203:143-181, November 2016.

D. Savoie. La gnomonique. Âne d'or. Les Belles Lettres, 2007.

S. E. Urban and P. K. Seidelmann. Explanatory Supplement to the Astronomical Almanac (3rd Edition). volume 223 of American Astronomical Society Meeting Abstracts, page 247.20, January 2014. 


\begin{tabular}{|c|c|c|c|c|}
\hline Hour (UT) & $H(\mathrm{deg})$ & $A(\mathrm{deg})$ & $h$ (deg) & $l(\mathrm{~mm})$ \\
\hline 3: 00 & -83.883 & -70.181 & 4.240 & 395.4 \\
\hline 3: 20 & -79.018 & -69.606 & 8.794 & 193.7 \\
\hline 3: 40 & -74.152 & -68.885 & 13.330 & 129.3 \\
\hline 4: 00 & -69.287 & -68.004 & 17.842 & 97.4 \\
\hline 4: 20 & -64.422 & -66.940 & 22.322 & 78.1 \\
\hline 4: 40 & -59.557 & -65.665 & 26.764 & 65.0 \\
\hline 5: 00 & -54.691 & -64.142 & 31.158 & 55.6 \\
\hline 5: 20 & -49.826 & -62.322 & 35.490 & 48.5 \\
\hline 5: 40 & -44.961 & -60.138 & 39.743 & 42.8 \\
\hline 6: 00 & -39.852 & -57.361 & 44.100 & 37.9 \\
\hline 6: 20 & -35.230 & -54.315 & 47.914 & 34.2 \\
\hline 6: 40 & -30.365 & -50.420 & 51.759 & 31.0 \\
\hline 7: 00 & -25.500 & -45.640 & 55.370 & 28.2 \\
\hline $7: 20$ & -20.635 & -39.758 & 58.666 & 25.9 \\
\hline 7: 40 & -15.769 & -32.557 & 61.535 & 24.0 \\
\hline 8: 00 & -10.904 & -23.886 & 63.838 & 22.5 \\
\hline 8: 20 & -6.039 & -13.806 & 65.413 & 21.6 \\
\hline 8: 40 & -1.174 & -2.737 & 66.116 & 21.2 \\
\hline 9: 00 & 3.691 & 8.549 & 65.872 & 21.3 \\
\hline 9: 20 & 8.557 & 19.188 & 64.706 & 22.0 \\
\hline 9: 40 & 13.422 & 28.573 & 62.738 & 23.2 \\
\hline 10: 00 & 18.287 & 36.482 & 60.125 & 24.9 \\
\hline 10: 20 & 23.152 & 42.982 & 57.021 & 27.0 \\
\hline 10: 40 & 28.018 & 48.272 & 53.552 & 29.6 \\
\hline 11: 00 & 32.883 & 52.575 & 49.812 & 32.6 \\
\hline 11: 20 & 37.748 & 56.099 & 45.870 & 36.1 \\
\hline 11: 40 & 42.613 & 58.979 & 41.778 & 40.4 \\
\hline 12: 00 & 47.479 & 61.371 & 37.570 & 45.5 \\
\hline 12: 20 & 52.344 & 63.361 & 33.273 & 51.9 \\
\hline 12: 40 & 57.209 & 65.024 & 28.907 & 60.1 \\
\hline 13: 00 & 62.074 & 66.416 & 24.486 & 65.8 \\
\hline 13: 20 & 66.940 & 67.581 & 20.022 & 86.9 \\
\hline 13: 40 & 71.805 & 68.551 & 15.523 & 111.5 \\
\hline 14: 00 & 76.670 & 69.350 & 10.997 & 155.9 \\
\hline
\end{tabular}

Table 2: Values of hour angle, azimuth, altitude and shadow length for a position of $4.5^{\circ} \mathrm{N}, 137^{\circ} \mathrm{E}$ on December 19 2018. Shadow length is computed for a gnomon of $28.7 \mathrm{~mm}$ high. 